\title{
Anabases
}

ANABASES Traditions et réceptions de l'Antiquité

12 | 2010

Varia

\section{La storia antica e la cultura classica}

\section{Emilio Gabba}

\section{OpenEdition}

\section{Journals}

Edizione digitale

URL: http://journals.openedition.org/anabases/1171

DOI: 10.4000/anabases. 1171

ISSN: 2256-9421

\section{Editore}

E.R.A.S.M.E.

\section{Edizione cartacea}

Data di pubblicazione: 1 ottobre 2010

Paginazione: 127-135

ISSN: 1774-4296

\section{Notizia bibliografica digitale}

Emilio Gabba, «La storia antica e la cultura classica », Anabases [Online], 12 | 2010, Messo online il 01 octobre 2013, consultato il 20 octobre 2019. URL : http://journals.openedition.org/anabases/1171 DOI : 10.4000/anabases. 1171 


\section{La storia antica e la cultura classica ${ }^{1}$}

Emilio GabBa

Sono molto legato alla città di Napoli e non posso dimenticare il periodo di studi che ho trascorso come allievo dell'Istituto italiano per gli Studi Storici nell'anno accademico 1949/50. Ho infatti potuto sentire non solo le lezioni indimenticabili di Benedetto Croce ma anche il corso di Storia Moderna tenuto da Federico Chabod, direttore dell'Istituto, e quello di Storia Antica del Prof. Pugliese Carratelli, recentemente scomparso, che fu poi mio giudice nel concorso universitario del ' 58 , sino alla morte ben vivo e presente nella mia attività scientifica, al cui ricordo rivolgo un sentito pensiero. Naturalmente non posso non ricordare gli altri miei colleghi, Ettore Lepore e Marcello Gigante, purtroppo scomparsi, ed altri colleghi ancora attivi nella ricerca scientifica, Girolamo Arnaldi, Giuseppe Giarrizzo e Gennaro Sasso.

L'argomento generale che mi propongo di trattare è molto vasto. Per questo ho pensato di ritagliare una riflessione sullo svolgimento degli studi di storia antica in Italia, con un'attenzione particolare alla situazione politico-culturale italiana ed europea. A partire dalla storiografia erudita di Carlo Sigonio fino ad arrivare all'opera di Muratori, si assiste al superamento della storia antiquaria, distinzione non facile, benché tenuta ben presente in Momigliano, e finita per essere proiettata sul mondo antico. Si pensi, infatti, al caso dell'indagine storico-antiquaria di Varrone di fronte alla vera e propria storiografia politica.

$1 \quad$ Si pubblica qui la stesura finale della lezione tenuta a Napoli il 23 maggio 2009 all'Istituto Italiano per gli Studi Filosofici per la Giornata Nazionale della Cultura Classica. Ringrazio l'amico Arnaldo Marcone per la predisposizione del testo. 
Successivamente in età illuministica, si sviluppa un dibattito sulla credibilità della storia romana arcaica e sull'attendibilità storico-letteraria, che può certamente considerarsi un inequivocabile segno di rinnovamento nella ricerca. Illustri esponenti della «storiografia filosofica», come Montesquieu nelle Considérations e nello Esprit des Lois, ma anche altri grandi nomi di questo periodo, da Ferguson a Vico e a Gibbon, la cui Storia del declino del mondo romano è in parte connessa col rapporto tra Impero e Cristianesimo, iniziano ad interrogarsi sul valore della narrazione antica, la quale non può essere supinamente accettata, ma deve essere attentamente vagliata. Nel tentativo di abbandonare un'indagine di tipo antiquario ed erudito ha così inizio una riflessione sui testi antichi, senza però giungere a quella che può essere definita una storiografia critica. A ciò si aggiunga la grande influenza della scoperta di Ercolano e di Pompei, la quale ha introdotto nuovi elementi nel dibattito ormai di carattere epistemologico. Il dato archeologico mette sulla scena una realtà storica concreta con cui la realtà storica deve confrontarsi. Accenno semplicemente alla vasta e complessa opera del De Brosses, di cui mi ero occupato in tempi passati, legata sia alle scoperte archeologiche ${ }^{2}$, sia alla storia della fine della Repubblica romana ${ }^{3}$, studiata soprattutto su Sallustio. Apro ora una piccola parentesi per introdurre una singolare questione, che non è possibile qui trattare, ovvero la possibile trasmissione orale di notizie storiche; per la storia romana il problema parte da Catone ed arriva almeno a Niebuhr, ed ha possibilità di essere identificato in alcuni momenti molto curiosi.

Alla fine dell'età illuministica si è avuto il passaggio alla ricostruzione critica della storia, per quella romana con le opere di Niebuhr, le cui traduzioni in lingua italiana sono compiute agli inizi dell'Ottocento a Pavia ${ }^{4}$ e a Napoli, e per il diritto romano con Savigny. Il tema della formazione e dell'organizzazione dello stato diviene centrale nell'esercizio dell'indagine storica del XIX secolo. Dai testi antichi si vuole comprendere in che modo una comunità di semplici persone possa diventare una comunità di cittadini. Echi di tale impostazione si trovano nella Römische Geschichte di Mommsen, con un decisivo intervento della testimonianza epigrafica ed archeologica. E' bene precisare che ogni ripensamento di natura storica, anche nella storiografia ottocentesca, si lega indelebilmente con le vicende contemporanee. Per esempio, nel periodo della Restaurazione, lo storico e politico francese, François Guizot, riprese la valorizzazione, o, per meglio dire, lo studio e il significato delle libertà municipali antiche ${ }^{5}$. Egli si rese

2 Ch. De Brosses, Lettres sur l'état actuel de la ville souterraine d'Herculée et sur les causes de son ensevelissement sous les ruines du Vésuve, Dijon, 1750.

3 Ch. De Brosses, Histoire de la République Romaine dans le cours du vile siècle, par Salluste; en partie traduite du latin sur l'original; en partie rétablie et composée sur les fragments qui sont restés de ses livres perdus, Dijon, 1777.

4 M.B.G. Niebuhr, Storia Romana, Pavia, 1832.

5 Fr. Guizot, “Du régime municipal dans l'Empire romain, au v viècle de l'ère chrétienne, lors de la grande invasion des Germains en Occident", in Essais sur l'histoire de la France, Paris, 1841, p. 6-39. 
conto che queste libertà rappresentavano spazi di partecipazione politica per i cittadini di fronte alla diminuzione di quella nel centro dello Stato con la formazione dell'Impero. L'età imperiale è un momento di declino di queste libertà municipali, sviluppatesi in Italia dopo la Guerra Sociale ${ }^{6}$, il quale coinvolge le classi medie, come la questione della centralizzazione amministrativa in Francia chiama in causa la media borghesia, protagonista della scena politica dopo la Costituzione del 1814.

Se, come abbiamo poco sopra affermato, Niebuhr può considerarsi l'iniziatore del ripensamento critico della storia di Roma, l'Italia riceve con l'opera di Ruggero Bonghi quello che ancora le mancava. La sua Storia di Roma (tre volumi, i primi due editi fra il 1884 e il 1888 , e l'ultimo postumo del $1896^{7}$ ) è stata spesso svalutata, perché non era un testo proveniente dagli ambienti accademici. In realtà è un interessante ripensamento politico, con un'ottima conoscenza bibliografica, della storia arcaica di Roma come momento fondamentale della formazione dello stato romano. Egli traccia una linea diretta tra l'esperienza romana e quella della formazione degli stati italiano e germanico nella seconda metà dell' $800^{8}$. Questo si giustifica col fatto che Bonghi era un acuto uomo politico, con precisi interessi concreti che ricadevano sule analisi della tradizione antica: egli infatti è stato relatore della Legge delle Guarentigie dopo il 1870. Il suo lavoro su Appio Erdonio ${ }^{9}$, ricordato con grandi elogi da Münzer nella Realencyclopädie di Pauly-Wissowa ${ }^{10}$, non aveva ricevuto nessun apprezzamento da parte degli storici accademici italiani, perché l'autore non era considerato uno specialista.

Chi riconobbe il sua valore, e questo già di per sé è un merito altissimo, fu Karl Julius Beloch che gli dedicò l'Italische Bund ${ }^{11}$ e fu suo successore sulla cattedra di

6 Il problema delle libertà municipali, fondamentale per la storia italica del I secolo a.C., ha origine con la Guerra Sociale. Per un'analisi più dettagliata degli aspetti riguardanti la concessione della cittadinanza romana agli alleati italici e la creazione del sistema municipale nella penisola rinvio ai miei studi Esercito e società nella tarda Repubblica romana, Firenze, 1973 e Italia romana, Como, 2000.

7 Sulle vicende che portarono alla pubblicazione di questo testo si rinvia al mio contributo, "La 'Storia di Roma' di Ruggro Bonghi”, in E. GABBA, Cultura classica e storiografia moderna, Bologna, 1994, p. 161-182.

8 Nel primo volume, I re e la Repubblica sino all'anno 283 di Roma, si trova una lettera in cui l'autore esplicitamente connette passato e presente: all'unificazione dell'Italia raggiunta con la forza si contrappone quella realizzata in modo ben diverso nell'età contemporanea. E' ovvio che la storia di Roma venisse intesa in chiave risorgimentale unitaria ed è questa una tendenza che ha influenzato a lungo lo studio della storia antica in Italia, come si può leggere nel presente testo a proposito di Gaetano De Sanctis.

9 R. Bonghi, "Appio Erdonio. Critica di critica”, in Nuova Antologia, II s., 19 (1880), p. 399-442.

10 Pauly-Wissowa, Realencyclopädie, s.v. Herdonius, col. 619-620.

11 Der Italische Bund unter Roms Hegemonie. Staatsrechtliche und Statistische Forschungen, Leipzig, 1880. 
Roma. La sua opera si colloca in una fase della ricerca che possiamo definire positivistica. Il centro degli interessi, che continua nei primi vent'anni del secolo ventesimo, non è tanto la storia della cultura, ma l'economia, la popolazione, l'organizzazione giuridico-politica e la colonizzazione. L'attenzione deve rivolgersi alle cose concrete, territori, popolazioni, forze militari, capacità economiche e commerciali degli stati. Vi è quindi il tentativo di rompere con una tradizione storiografica che aveva privilegiato la narrazione della storia politica, militare ed amministrativa, per entrare nel concreto di una realtà storica da comprendere e valutare nei suoi dati quantitativi. Per questo la ricostruzione degli ambiti territoriali dello stato romano e delle città italiche è condotta sulla base delle informazioni epigrafiche, storico-letterarie e sui confini delle diocesi, che riproducevano alle loro origini quelle dei municipi romani. Inoltre la quantificazione di quegli ambiti, ottenuta attraverso tecniche statistico-matematiche, deve servire some base concreta entro la quale collocare i dati numerici demografici, a loro volta calcolati secondo criteri euristici precisi.

Questo metodo di ricerca, rozzo ma efficace, proprio per l'avvalersi di metodologie impiegate nella raccolta e nello sfruttamento di dati per esigenze politiche e scientifiche contemporanee, dava l'impressione (alcuni direbbero l'illusione) di una maggiore scientificità, rigore e oggettività in quanto serviva a stabilire rapporti tra il territorio, popolazione, produzioni, forze militari e capacità economiche. Non vi è dubbio che l'aspetto statistico della ricerca ha un ruolo prevalente nel quadro complessivo rispetto a quello giuspubblicistico pur ricordato nel titolo.

Una posizione a sé stante è quella iniziale di Ettore Pais (1856-1939). Lo studioso $^{12}$ affronta la storia di Roma nei prime cinque secoli come raccolta e analisi dei materiali letterari e documentari in una prospettiva in definitiva non dissimile da quella che era stata la critica settecentesca, ma con l'applicazione di una metodologia filologica spinta alle estreme conseguenze: essa è una critica della tradizione più che una ricostruzione dello svolgimento storico, che verrà in una seconda fase ${ }^{13}$. Il merito di Pais fu quello di ribadire il valore politico della riflessione storiografica, considerando la storia d'Italia come momento fondante della storia unitaria italiana. Questa visione gli fu probabilmente suggerita dall'approfondito studio della storia siciliana, letta come contrasto fra l'elemento greco ed elemento punico, con la conseguente unità della storia della Sicilia e della Magna Grecia. Egli arriva a concepire Roma come erede di Siracusa nella lotta contro Cartagine e, sottolineando la vastissima presenza di tradizioni storiche e storiografiche greche nella storia di Roma arcaica, segnala le antichissime connessioni tra Magna Grecia, Sicilia e Roma.

Questa interpretazione unitaria della storia d'Italia (della quale elemento centrale era la storia di Roma repubblicana che aveva realizzato l'unificazione politica della

12 E. PAIS, "Storia di Roma", I-1-2 in Storia d'Italia. Dai tempi più antichi sino alle guerre puniche, Torino, 1898-1899.

13 E. PAIS, Storia di Roma durante i primi cinque secoli, Roma, 1913. 
penisola) doveva naturalmente essere caratteristica della storiografia italiana, la quale nascondeva il germe di una connotazione nazionalistica della storia. Questo atteggiamento va accentuandosi nello sviluppo storiografico del Pais, tanto che, verso la fase finale della sua vita, egli venne ad assumere, più che altro a parole, atteggiamenti conformistici verso il fascismo, che furono facilmente riverberati anche sulla sua precedente attività.

Questa singolare personalità di storico, che dominò i decenni fra XIX e XX secolo nell'antichistica non soltanto italiana, fu a capo di una scuola di studiosi di alto valore, influenti nel mondo scientifico e accademico fin oltre la metà del '900. Il principale allievo di Pais fu Emanuele Ciaceri, che studiò con metodo sicuro e largo sguardo la Magna Grecia e Cicerone, di cui scrisse una biografia ${ }^{14}$. Ricordo inoltre Ettore Ciccotti, inviato dal Ministero a Pavia nell'anno accademico 1897-1898 alla Facoltà di Lettere. Il suo trasferimento dall'Accademia scientifico-letteraria di Milano fu quasi forzato, perché la Facoltà era contraria e solo tre furono i voti favorevoli. Impegnato politicamente su posizioni di sinistra, fu coinvolto nei fatti di Milano nel 1898, e perciò gli fu negata la conferma ${ }^{15}$ sulla cattedra pavese. Interessato a problemi soprattutto di storia della società ed economici, aveva scritto dei lavori notevoli sulla storia della schiavitù ${ }^{16}$, tema molto dibattuto nella ricerca occidentale, in Francia, Gran Bretagna, Germania e negli Stati Uniti. Il suo orientamento storiografico rappresentava perciò una forma di materialismo storico abbastanza avanzata per l'Italia. Aveva goduto per questi suoi indirizzi scientifico-letterari una giusta rinomanza nel mondo non solo accademico italiano.

Di poco posteriore a Pais, il cattolico Gaetano De Sanctis, allievo di Beloch, fu professore a Torino dal 1900 al 1929. Non giurò fedeltà al fascismo nel 1931, perdendo così la cattedra, che riprese nel 1944 all'Università di Roma, ove insegnò fino alla morte nel 1957. Il De Sanctis pose sempre come criterio interpretativo della storia greca il concetto secondo cui l'unità politica fu sempre l'aspirazione comune e concorde e la ragione degli stati greci. Egli rimase ben fermo nella distinzione fra unità politica e unità culturale, giacché la pur riconosciuta consapevolezza da parte del mondo greco di una cultura unitaria - onde può darsi una storia della civiltà greca che può esistere senza l'unità politica - non gli appariva criterio sufficiente per impostare il problema. Anche se questa unità non ha portato alla creazione di una vera e propria struttura sociale, essa rappresenta un fatto determinante della storia greca, indipendentemente dalle diverse forme di organizzazione politica susseguitesi nel tempo, delle poleis allo stato federale, dalle egemonie agli stati ellenistici. Da ciò si spiega anche il giudizio negativo verso ogni tentativo di sopraffazione politica esterna del mondo greco, in

14 E. Ciaceri, Cicerone e i suoi tempi, I-II, Milano, 1926-1930.

15 G. Manganaro Favaretto, Ettore Ciccotti (1863-1939): il difficile connubio tra storia e politica, Trieste, 1989.

16 E. Cіссотті, Il tramonto della schiavitù nel mondo antico, Torino, 1899. 
particolare contro Roma. Egli attribuisce al dominio romano la decadenza del mondo greco e naturalmente l'interruzione del movimento unitario, che avrebbe a suo dire vigoreggiato prima dell'intervento di Roma. Ha parole sprezzanti verso i greci amici, o fautori dei Romani, dei quali l'esempio più illustre è Polibio. De Sanctis studiò l'organizzazione politica della comunità romana, poi dello stato e del sistema italico (alleanze, federazione, tendenze unitarie), le guerre unificatrici (Pirro e Cartagine), mostrandosi contradditoriamente ostile ad una politica imperialistica che si rivolgesse verso il mondo ellenistico, ma favorevole a quella verso l'Occidente gallico, ispanico e cartaginese: Cartagine era considerata un peso morto per la civiltà classica. De Sanctis è l'autore della Storia dei Romani, che fino al volume III è una ragionata ricostruzione critica della tradizione, della storia e della cultura di Roma, dalla preistoria sino alla Guerra Annibalica; nel IV volume si percepisce l'influenza del pensiero storiografico di Croce, specialmente nell'idea della storia come idea di libertà, di qui la famosa dedica del 1924: "A quei pochissimi che hanno parimenti a sdegno d'essere oppressi e farsi oppressori ${ }^{17}$." In questo cambio di impostazione si percepisce l'eco delle vicende contemporanee: il volume fu scritto all'indomani della prima guerra mondiale e nel mezzo delle convulsioni politiche e sociali che l'Italia viveva alla fine degli anni venti, con l'inizio del regime fascista. La riflessione su concetto della libertà greca che coinvolse Momigliano e Ferrabino, allievo del De Sanctis, che, contro le tendenze nazionali greche del suo maestro, aveva sviluppato il tema dello spirito federale.

Accanto alle vaste ricerche di Momigliano, sul cui storicismo ho già scritto ${ }^{18}$, un altro allievo del De Sanctis, Mario Attilio Levi, studiò i modi dell'affermazione del potere alla fine della Repubblica (Silla ed Ottaviano). Della sua scuola ricordo infine Luigi Pareti, che potei ascoltare qui a Napoli nel 1949-50, storico dotato di larghissimo sguardo su tutta l'Antichità, con grande capacità critica e maestro di Santo Mazzarino.

Dedico ora brevi cenni all'indirizzo di ricerca di Plinio Fraccaro, al quale anch'io mi onoro di appartenere. Ho già scritto di lui più volte ${ }^{19}$, sicché sono, credo, autorizzato a limitarmi a una breve sintesi. Professore di Storia Antica dal $1915^{20}$

17 G. De Sanctis, Storia dei Romani, IV, 1, Torino, 1923.

18 E. GabBA, "Aspetti della storiografia di Arnaldo Momigliano", Rivista Storica Italiana 100 (1988), p. 361-380; "Ricordo di Arnaldo Momigliano storico del mondo antico", in GABBA, Cultura classica, p. 393-402.

19 E. GabBa, "Plinio Fraccaro", in GabBA, Cultura classica, p. 371-378.

20 Fraccaro aveva vinto il concorso nel 1915 con molta difficoltà, a causa di opposizioni non tanto della commissione, quanto dei candidati sconfitti, che cercarono in vario modo di compromettere anche la vittoria di Fraccaro. Infatti per il primo posto Fraccaro ebbe quattro voti; per il secondo gli altri candidati ebbero un voto ciascuno; la mancata composizione della terna provocò la proposta di annullamento del concorso, che si concluse verso la fine del 1916 con la decisione favorevole alla nomina di Fraccaro. Lo svolgimento del concorso viene descritto in due contributi di Leandro Polverini dedicati 
al 1953 all'Università di Pavia, di cui divenne rettore ${ }^{21}$, Fraccaro si era laureato a Padova con Antonio Cima ${ }^{22}$. La sua tesi di laurea, che ricostruiva l'opera perduta di Varrone De gente populi Romani, fu pubblicata nel $1907^{23}$. Seguendo poi l'indirizzo del suo maestro, si era messo a studiare l'oratoria romana soprattutto nel III-I secolo a.C. e, dallo studio dell'oratoria romana, aveva fermato la sua attenzione sulla figura centrale di Catone. Naturalmente lo studio dei frammenti degli oratori, intesi come massima espressione dell'attività politica, comportava il passaggio all'analisi della politica, dell'amministrazione dello stato e della struttura della società. Grazie alle opere degli oratori, potevano essere studiati l'organizzazione della vita politica di Roma fra il III e il II secolo secolo a.C., i rapporti fra politica e esercito nonché vari aspetti del sistema giuridico pubblico e privato. Per questo importanti sono i lavori dedicati al diritto pubblico, i quali corressero in molti punti il sistema di Mommsen e, per questo argomento, rinvio, nel fascicolo degli atti di Bassano, al bellissimo saggio di Dario Mantovani ${ }^{24}$.

La sua opera si è venuta ampliando, sempre sulla base dell'oratoria, a uno studio complessivo dei fenomeni politici dell'imperialismo romano. L'attenzione di Fraccaro andò molto a fondo negli aspetti giuridico-amministrativi, oltre che politici, e la sua opera su Catone rappresentò e rappresenta un'interpretazione della storia di Roma nell'età tardorepubblicana che è praticamente insostituibile. Voglio perciò precisare un punto: egli si accorse subito che per ricostruire la storia politico-amministrativa dell'espansione di Roma in Italia e nelle aree mediterranee era indispensabile afferrare sempre meglio l'organizzazione del territorio, attraverso uno studio storico dell'ambiente, considerato nella sua realtà umana, oltre che ovviamente economica e culturale. Da questa interpretazione storica del territorio in cui i fatti politici si verificano, derivò una sua considerazione dell'archeologia non solo come dato monumentale, legato ai resti più o meno evidenti e conservati della vita antica, ma propriamente come una testimonianza della realtà sociale e del contesto in cui questa vita antica si svolgeva. Di qui nasce una serie di lavori fondamentalissimi: Fraccaro fu l'iniziatore degli studi in Italia sull'organizzazione del territorio, la cd. centuriazione, e sul suo rapporto con l'urbanizzazione, con la rete viaria e fluviale. Molte tesi da lui dirette riguardavano la storia di città, soprattutto transpadane, vecchie e nuove. Il suo interesse per le fasi preromane,

a Fraccaro ("Fraccaro e De Sanctis", Athenaeum 73 [1985], p. 68-113; "Dal carteggio di Plinio Fraccaro", Athenaeum 83 [1995], p. 411-429).

21 Fraccaro fu il primo rettore ad essere nominato dalla caduta del regime fascista dal Governo Militare Alleato, poi confermato dal consenso dei colleghi fino al giorno della sua scomparsa.

22 P. Treves, "Cima Antonio", in Dizionario Biografico degli Italiani, XXV (1981), p. 516-519.

23 P. Fraccaro, Studi varroniani. De gente populi Romani libri IV, Padova, 1907.

24 D. Mantovani, "Plinio Fraccaro e il Diritto pubblico romano", in Atti della Giornata in ricordo di Plinio Fraccaro, Athenarum 89 (2001), p. 47-72. 
celtiche, liguri e venete, attestate anche nella toponomastica, era vivissimo. La lettura delle carte topografiche e della loro interpretazione non si fermava, del resto, alla realtà antica, ma si svolgeva in una proiezione molto avanzata. D'altra parte, il Fraccaro, come ho detto in un saggio recente ${ }^{25}$, aveva trasmesso ai suoi allievi una visione storica più ampia, anche se la sua attenzione era stata incentrata prevalentemente sulla storia romana. Fino al 1935 il suo insegnamento aveva come titolatura "Storia antica» e solo poi fu cambiata per motivi anche accademico-politici in «Storia greca e Storia romana con esercitazioni di epigrafia romana». Per Fraccaro, tuttavia, era sempre rimasto valido il concetto di storia antica. Questo vuol dire che egli aveva sempre nutrito un interesse molto vivo per tutte le forme della civiltà che confluivano intorno al Mediterraneo, perciò non solo la storia greca, ma anche la storia del Vicino Oriente. Fraccaro era rimasto molto colpito dalla «scoperta» della civiltà etea (così secondo il testo biblico), cioè degli Ittiti, in Anatolia; aveva attentamente studiato questa fase storica anche grazie alle numerose testimonianze pervenute, come le iscrizioni rupestri, e aveva così spronato alcuni allievi a continuare tali ricerche. Fondatore di una scuola rigogliosa, ha avuto come allievi Gianfranco Tibiletti, Alfredo Passerini, Aurelio Bernardi ed Enrica Malcovati, che hanno proseguito il suo indirizzo di studi in maniera unitaria, pur sviluppando personali filoni di ricerca.

Da Ferrabino deriva Franco Sartori con i suoi lavori di Storia Greca e Romana. Così dalle ricerche scientifiche svolte in Napoli e dalle tendenze culturali e filosofiche di ambiente crociano discendono tanto il pensiero storiografico di Giovanni Pugliese Carratelli, che è anche un illustre epigrafista, quanto la riflessione storica di Ettore Lepore del quale ho avuto la ventura di veder svolgere il complesso ragionamento sul Princeps ciceroniano ${ }^{26}$. Aveva una mente di grande sensibilità e capacità critica $\mathrm{e}$ ricchezza di problemi filosofico-culturali.

Come ho già accennato più sopra, è stato allievo del Pareti a Catania e ha ripreso anche dal maestro la grande varietà e fantasia nella ricostruzione storica Santo Mazzarino, che ha spaziato su tutta la storia e la storiografia antiche. Voglio qui considerare soltanto il suo grande Pensiero storico classico $^{27}$ con il superamento della distinzione fra antiquaria e storiografia e l'interpretazione storica di ogni forma letteraria antica, nonché la sua visione del cattolicesimo tardoantico come democrazia, in quanto diffusione di cultura che ha eclissato le tradizionali forme elitarie della cultura e della letteratura antica.

25 E. GabBa, "Sull'insegnamento di Plinio Fraccaro all'Università di Pavia. Ritratti di Maestro e allievi”, Athenaeum 97 (2009), p. 229-239.

26 E. Lepore, Il Princeps ciceroniano e gli ideali politici della tarda Repubblica, Napoli, 1954.

27 S. Mazzarino, Il pensiero storico classico, Bari, 1966. 
Ho riletto in questi giorni alcune pagine del libro di J. Diamond, Armi, acciaio e malattie. Breve storia del mondo negli ultimi tredicimila anni ${ }^{28}$; ho così concluso che non posso certo dubitare delle nefandezze che gli esseri umani hanno compiuto nello stravolgere la natura del pianeta. Resta tuttavia il mistero del perché l'uomo sia stato collocato nello sterminato universo, a quel che sembra, solo sulla terra. Ma per noi non è un mistero che nei tempi storici, pur nelle tragedie delle quali siamo stati e siamo testimoni, si sia venuta sviluppando una consapevolezza morale, legata ad una tradizione di cultura, della quale anche oggi siamo orgogliosi.

Emilio GABBA

Professore emerito di Storia Antica

dell'Università di Pavia

Via Maggi 2

I - 27100 Pavia

28 J. Diamond, Armi, acciaio e malattie. Breve storia del mondo negli ultimi tredicimila anni, Torino, 2000. 